\title{
PRESENTACIÓN
}

\section{Entender la violencia}

\author{
Elena Azaola
}

$\mathrm{E}$ 1 texto Los vicios ordinarios de la conocida politóloga Judith Shklar explica que pocos filósofos se han dado a la tarea de estudiar a fondo la crueldad por lo que, para aproximarse a esta manifestación extrema, que nos enfrenta con nuestra propia irracionalidad como ninguna otra conducta, es necesario acudir a escritores, poetas y dramaturgos de la antigüedad. Shklar encuentra que para Montaigne era preciso colocar a la crueldad en primer lugar entre los vicios ordinarios, pero nos advierte que si contemplamos - especialmente durante un periodo prolongado- la deshonestidad, la infidelidad y la crueldad, nos volveremos misántropos, como le ocurrió al propio Montaigne. La misantropía, nos dice, tiene los efectos políticos más corrosivos. Si uno coloca la crueldad primero, debe tener cuidado de controlar la propia misantropía, que puede convertirse en furia (Shklar, 1984).

En efecto, la crueldad como una de las formas de expresión de la violencia extrema constituye una especie de enigma, que al mismo tiempo que nos atrae nos horroriza. Intentamos comprenderla, las más de las veces en vano, quizá con el propósito de someterla a control o evitarla si fuese posible. Ante la crueldad difícilmente podemos permanecer indiferentes. Se trata de una de las manifestaciones posibles del accionar humano que nos reta, nos interpela y que buscamos situar en un horizonte de inteligibilidad, en un universo de sentido, quizá con la pretensión de que al entenderla podamos mantenerla a raya y someter aquello que nos repele, que nos duele y nos atemoriza...

Este número de Desacatos, uno más que intenta aproximarse al fenómeno de la violencia desbordada en nuestro país, no pretende resolver las múltiples interrogantes ni allanarnos el camino u ofrecernos una vía rápida para llegar a ese horizonte de inteligibilidad al que anhelamos acceder. Pretende, solamente, explorar determinados ángulos desde los que nos parece que ciertas expresiones de la violencia pueden ser mejor entendidas. Aspira, asimismo, a participar de manera modesta en el debate que en los últimos tiempos ha ocupado, y en ocasiones obsesionado, a los especialistas en seguridad, que una y otra vez han intentado responder a la pregunta: ¿cómo es posible que la violencia explotara de modo tan

\section{Understanding Violence}

Elena Azaola: Centro de Investigaciones y Estudios Superiores en Antropología Social-Distrito Federal, México eazaola@ciesas.edu.mx

Desacatos, núm. 40, septiembre-diciembre 2012, pp. 7-10 
abrupto como lo ha hecho durante los últimos cinco años en nuestro país? ${ }^{1}$

Tal vez este debate no ha encontrado respuestas que generen consenso porque todavía se encuentra demasiado impregnado o contaminado por los argumentos que esgrimen las distintas fuerzas políticas. Y, como sabemos, los argumentos políticamente motivados suelen tener una perspectiva más bien estrecha, si no es que deliberadamente sesgada. En contraste, si uno mira lo que se ha producido desde las ciencias sociales durante las últimas décadas, se encuentran pocos estudios que hubieran elegido como objeto la violencia, sin embargo, una buena cantidad se topó con ella y aportó información valiosa. Desafortunadamente, esta información no ha sido sistematizada ni analizada a la luz de la situación y de la descomposición brutal que ha tenido lugar en zonas cada vez más extensas de nuestro país. Aunque sus aportes están todavía por ser recuperados, es claro que estos estudios cuentan, por lo general, con una mirada histórica más profunda y con un conocimiento más amplio y detallado de lo que ocurre a nivel local, en comparación con aquellos que casi de manera exclusiva han centrado su atención en las curvas de la violencia de la última década. ${ }^{2}$

La recuperación de los aportes que éstos y otros estudios han hecho para entender mejor la violencia en nuestro país es una tarea todavía por realizar y que, desafortunadamente, cae fuera de los límites tanto de esta presentación como de los que se han

\footnotetext{
${ }^{1}$ En estos debates han participado de manera destacada, entre otros, Fernando Escalante, Eduardo Guerrero, Joaquín Villalobos y Alejandro Hope, cuyos textos pueden consultarse en los números de la revista Nexos de 2008 a la fecha.

${ }^{2}$ Sin pretender hacer un listado exhaustivo pueden mencionarse los estudios realizados desde disciplinas diversas por Luis Astorga, Carlos Flores, Arturo Alvarado, Mario Arroyo, Roberto Castro, Juan Manuel Valenzuela, René Jiménez, Marcelo Bergman, Nelson Arteaga, Juan Cajas, Eduardo Menéndez, Aída Hernández, Clara Jusidman, Xochitl Leyva, Luciana Ramos, Guadalupe Rodríguez, Edna Jaime, Lucía Melgar, Julia Monarrez, Guilherme Borges, Francisco Pamplona, Rossana Reguillo, Witold Jacorzynski, Patricia Ravelo, Sergio Zermeño, Cristina Zubillaga y un largo etcétera que se disculpa por las no deliberadas omisiones.
}

trazado los trabajos que incluye este volumen, cuyo contenido resumo a continuación.

La sección principal de la revista contiene tres trabajos. Me referiré brevemente a ellos en orden de aparición sin intentar calificarlos - ya lo han hecho nuestros dictaminadores, en algunos casos con bastante rudeza- . Nos reservamos para la opinión que nuestros textos merezcan por parte de los lectores. Un primer artículo, del que soy autora, desarrolla tres argumentos para explicar los niveles de violencia que padecemos hoy en el país. El primero se refiere a la presencia de formas de violencia que han existido desde tiempo atrás, que no se relacionan directamente con las actividades de grupos delictivos y que han sido toleradas -incluso ignoradasy cuyos efectos acumulados a lo largo del tiempo han contribuido al actual escalamiento de la violencia. A estas formas de violencia las denomino las "violencias de siempre". El segundo argumento apunta al debilitamiento y la descomposición, que tampoco han recibido una respuesta apropiada por parte de las instituciones de seguridad y procuración de justicia, que cuentan con una escasa capacidad para investigar los delitos y procesar a los responsables, lo que ha traído como consecuencia un incremento de la impunidad, que a su vez ha propiciado un crecimiento exponencial de la criminalidad en general y de la violencia en particular. A esta forma de violencia la llamo la "violencia de hoy". El tercer argumento se dirige hacia las políticas sociales y económicas insuficientes que no han logrado reducir las desigualdades y promover la inclusión de amplios sectores de la población. A esta forma de violencia la denomino "violencia estructural" y planteo que, a pesar de haber sido normalizada, no deja de tener efectos que se manifiestan en los actuales niveles de violencia exacerbada en nuestro país.

El segundo texto, de Rossana Reguillo, intenta descifrar el lenguaje, la gramática de la violencia y desmenuzarla de manera similar a la que se emplea para destazar los cuerpos. Encuentra que tanto para los delincuentes como para los agentes del Estado parece necesario inscribir las huellas de su 


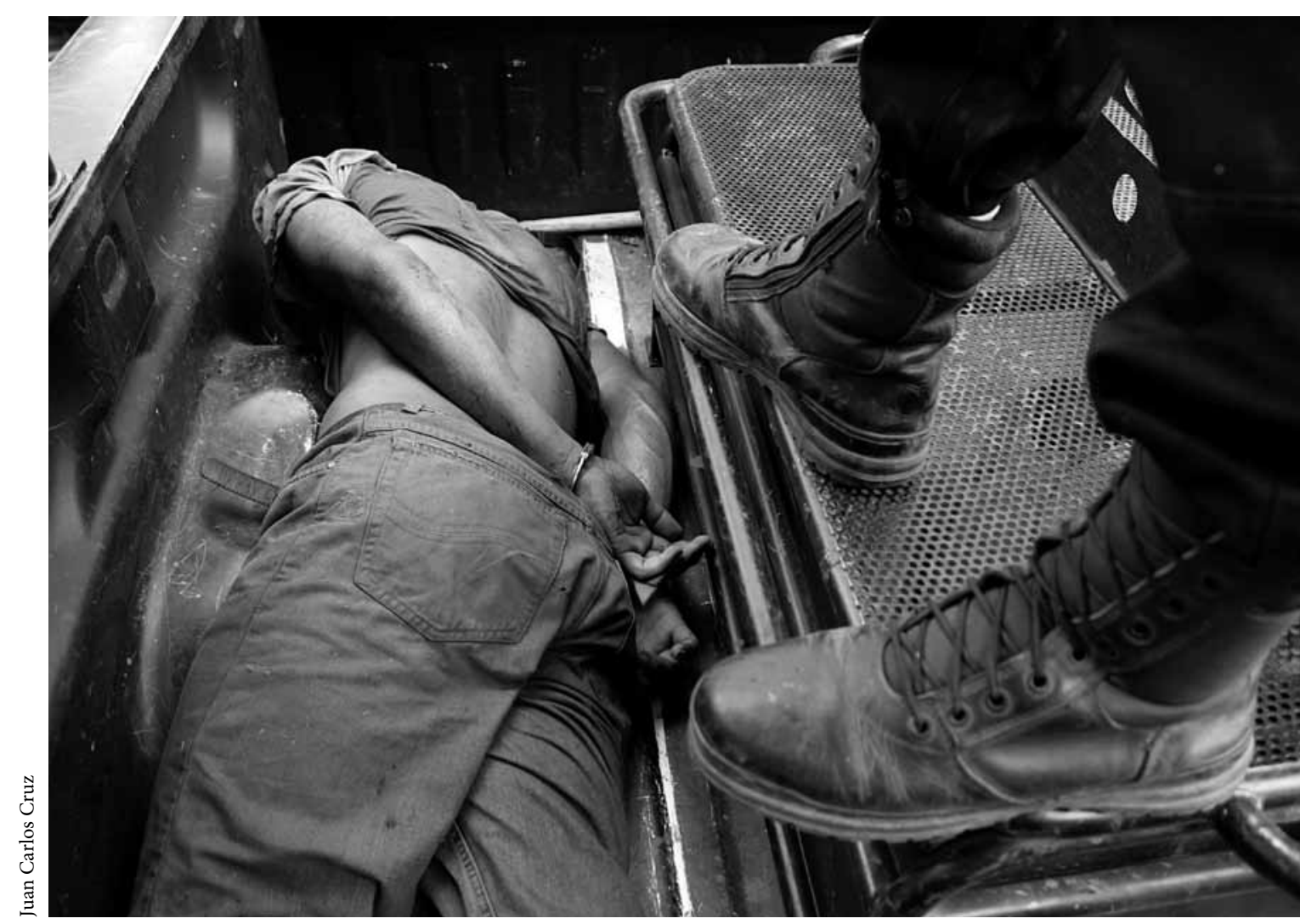

Un policía estatal vigila a uno de los supuestos sicarios detenidos después de un enfrentamiento en Navolato, Sinaloa, 2011.

poder sobre los cuerpos ya muertos, aunque también podríamos agregar a los de los detenidos o los desaparecidos. Reguillo se pregunta por las reglas, las pautas, los usos y los dispositivos de los hechos violentos. Establece que no es posible pensar y entender las violencias que derivan del narcotráfico al margen de las condiciones estructurales de la sociedad, donde cabe situar el proceso que denomina de "desafiliación acelerada" que ha tenido lugar entre los jóvenes. La autora desmenuza el signo de las violencias que se inscriben en el capitalismo tardío. Igualmente, y de manera inquietante, hace notar el desgaste que se observa en los símbolos del orden instituido.

Por su parte, el trabajo de Carlos Silva Forné, Catalina Pérez Correa y Rodrigo Gutiérrez Rivas aboga por la necesidad de conocer los resultados del uso y el abuso de la fuerza por parte de las fuerzas federales - Policía Federal, Ejército y Marina- en los enfrentamientos contra presuntos miembros de la delincuencia organizada. A través del análisis meticuloso de la información empírica disponible, los autores construyen un índice de letalidad que da cuenta de la proporción de muertos y heridos que han resultado de los enfrentamientos de presuntos delincuentes con cada una de las instituciones federales mencionadas. Discuten los resultados de sus hallazgos a la luz de los estándares relativos a la prevención de las ejecuciones extrajudiciales, arbitrarias o sumarias. El trabajo aporta elementos a considerar en el indispensable debate en torno a la autorización, o no, de la participación del Ejército y la Marina en tareas de seguridad pública y, de ser el caso, acerca de cuáles deben ser los límites que 
deben regir su actuación. Asimismo, colocan sobre la mesa la necesidad de contar con información confiable sobre el resultado del uso y el abuso de la fuerza por parte del Estado.

A los trabajos anteriores, que ofrecen un conjunto diverso de miradas y aproximaciones al tema de la violencia, se suma el balance y el comentario de Marcelo Bergman, que agrega y enriquece la discusión con sus propios puntos de vista respecto de los factores que, en su opinión, han contribuido a exacerbar la violencia en nuestro país. Subraya la necesidad de ir más allá de la actual coyuntura delictiva y de las políticas que intentan contenerla, para profundizar en las variables estructurales que subyacen a la violencia. Plantea que la epidemia delictiva no irrumpió, como a menudo se pretende hacer creer, de manera intempestiva, sino que se fue gestando a fuerza de "esconder los problemas bajo el tapete". Bergman analiza las tendencias delictivas de los últimos 80 años para sostener que hay que mirar más allá de la coyuntura actual, al tiempo que esboza algunas líneas de investigación que habrá que explorar para entender mejor de qué está hecha la violencia en nuestro país.

Por último, nos pareció que un número dedicado al análisis de la violencia debería aprovechar la sección “Testimonios" para dar voz a la expresión política más articulada que ha hecho visibles los profundos daños ocasionados por la violencia a las personas, las familias y las comunidades y ha hecho un llamado consistente para detenerlos. Incluimos la trayectoria del Movimiento por la Paz con Justicia y Dignidad, así como una entrevista a sus dos líderes más destacados: Javier Sicilia y Emilio Álvarez Icaza.

Esperamos que este número de Desacatos haya cumplido con el objetivo de proporcionar elementos que nos permitan ampliar y profundizar nuestra mirada sobre los preocupantes niveles de violencia que vive nuestro país. Lo hemos hecho con la convicción de que las ciencias sociales disponen de herramientas específicas que deben ser puestas al servicio de las tareas más urgentes que tiene frente a sí el país. Pero nuestras herramientas no deben servir sólo para desmenuzar la violencia o para comprender mejor los factores que han contribuido a exacerbarla, sino que también debemos utilizarlas para trazar el camino que habrá que recorrer para sanar y reparar las heridas, para participar en la reconstrucción de las comunidades más afectadas donde anhelamos que la vida pueda transcurrir con menos miedo, menos sobresaltos y menos dolor. De otro modo, corremos el riesgo de quedar atrapados, como nos advirtiera Judith Shklar, en nuestra propia misantropía.

\section{Referencias}

Shklar, Judith N., 1984, Ordinary Vices, Harvard University Press, Cambridge. 